\title{
Resveratrol inactivates PI3K/Akt signaling through upregulating BMP7 in human colon cancer cells
}

\author{
YU-HUA ZENG ${ }^{1,2^{*}}$, LIN-YUN ZHOU $^{1,2^{*}}$, QIAN-ZHAO CHEN ${ }^{1,2}$, YANG LI $^{1,2}$, YING SHAO $^{1,2}$, \\ WEN-YAN REN ${ }^{1,2}$, YUN-PENG LIAO ${ }^{1,2}$, HAN WANG $^{1,2}$, JIA-HUI ZHU ${ }^{1,2}$, \\ MING HUANG ${ }^{2}$, FANG HE ${ }^{2}, \mathrm{JIN}_{\mathrm{WANG}}{ }^{2,3}, \mathrm{KE} \mathrm{WU}^{1,2}$ and BAI-CHENG HE ${ }^{1,2}$ \\ ${ }^{1}$ Department of Pharmacology, School of Pharmacy, ${ }^{2}$ Chongqing Key Laboratory for Biochemistry and \\ Molecular Pharmacology, Chongqing Medical University, Chongqing 400016; ${ }^{3}$ Department of Blood Transfusion, \\ First Affiliated Hospital of the Chongqing Medical University, Chongqing 400042, P.R. China
}

Received November 23, 2016; Accepted May 11, 2017

DOI: $10.3892 / o r .2017 .5662$

\begin{abstract}
Colon cancer is common worldwide and accounts for the significant cancer related morbidity and mortality in patients. Although extensive advancement has been made in colon cancer treatment and diagnosis in the last decades, there is still a giant gap between the clinical expectation. It has been reported that resveratrol (Res) may be a potential candidate for cancer treatment. However, the specific mechanism underlying this activity remains unclear. In this study, we investigated the anticancer activity of Res in human colon cancer cells, and unveiled the possible mechanism for this effect. With cell viability, flow cytometry, PCR and western blot analysis, we demonstrated the efficacious anticancer activity of Res in HCT116 cells. Mechanically, we found that Res greatly upregulates BMP7 in HCT116 cells. Exogenous BMP7 enhances the anticancer effect of Res in HCT116 cells, which was almost reversed by the BMP7 specific antibody. Res does not activate the BMPs/Smads signaling, but decreases the phosphorylation of Akt1/2/3 substantially in HCT116 cells. Exogenous BMP7 enhances the inhibitory effect of Res on the phosphorylation of Akt1/2/3, while BMP7 immunodepletion reverses this effect notably. Res markedly decreases the phosphorylation of PTEN, which can be enhanced by exogenous BMP7 but partly reversed by the BMP7 antibody. Our findings suggested that Res may be a promising candidate for colon cancer treatment, and the anticancer activity may be mediated by inactivating
\end{abstract}

Correspondence to: Dr Bai-Cheng He or Dr Ke Wu, Department of Pharmacology, School of Pharmacy, Chongqing Medical University, 1 Yixueyuan Road, Yuzhong, Chongqing 400016, P.R. China

E-mail: 894704897@qq.com; hebaicheng99@yahoo.com

E-mail: wukecqmu@163.com

${ }^{*}$ Contributed equally

Key words: resveratrol, antiproliferation, colon cancer, BMP7, PI3K/Akt, PTEN
PI3K/Akt signaling through upregulating BMP7 to decrease, at least, the phosphorylation of PTEN.

\section{Introduction}

Colon cancer is the third most common cancer and the fourth cause for cancer related death globally. As reported, there are approximately 1.2 million new cases and 600,000 deaths per year (1). Depending upon the stage, the treatment of colon cancer involves multiple modalities, including surgery, radiotherapy, interventional radiology approaches and systemic chemotherapy. Although targeted therapy drugs for colon cancer have been introduced (2), such as bevacizumab, cetuximab and panitumumab, the prognosis remains unsatisfactory $(3,4)$. Thus, there is still a great clinical need to develop drugs for colon cancer treatment.

Traditional herb medicines or herbal derived components are one of the most widely used adjunctive therapies for cancer patients worldwide. The combination of these medicines with traditional chemotherapy drugs, such as camptothecin, vincristine and Taxol, plays an important role in cancer treatment (5-7). Res, a kind of non-flavonoid polyphenol, found in black grapes and red wine, is characterized as a phytoalexin and is produced by a variety of plants in response to stress. It was used as a natural plant compound in traditional Chinese and Japanese medicine $(8,9)$. Recently, it has been reported that Res can inhibit proliferation and promote apoptosis of various types of cancer cells, such as pancreas, breast and colon cancer (10-12). For colon cancer, although the anticancer effect of Res has been validated (12), the specific mechanism underlying this activity remains unclear.

Bone morphogenetic proteins (BMPs), one subgroup of transforming growth factor (TGF)- $\beta$ superfamily, play important roles in the regulation of multiple key steps of embryonic development, and the growth, differentiation and apoptosis of various cells. To date, $\sim 20$ members of the BMP subgroup have been identified (13). Recently, more evidence demonstrated that BMPs participate in cancer development and progression, such as colon cancer (14). It has been reported that BMP-9 promoted a proliferative response and exerted a remarkable anti-apoptotic function in hepatocellular carcinoma (HCC) 
cells (15), BMP-2 stimulated cellular proliferation in ovarian cancer cells (16). On the other hand, some data revealed the opposite role of BMP signaling in tumors. For instance, BMP-2 and BMP-7 function as effective tumor suppressors in gastric carcinoma, renal cell carcinoma, breast and colon cancer (17-19). BMP7 not only onsets its signaling through the canonical BMP/Smad pathway, but also the non-canonical BMP/Smad pathway, such as phosphatidylinositol 3-kinase (PI3K)/Akt (20,21). PI3K/Akt signaling plays a critical role in modulating cell survival and apoptosis (22), which has been found overactivated in many cancers to reduce apoptosis and promote proliferation. The tumor suppressor phosphatase and tensin homolog (PTEN) negatively regulates PI3K/Akt signaling and is often inactivated by mutations in a variety of cancer types.

To date, it remains unknown whether BMP7 is associated with the anticancer activity of Res in colon cancer cells. In the present study, we investigated the possible role of BMP7 in the antiproliferative effect of Res in HCT116 cells, and we elucidated the possible mechanism of how BMP7 exerts this function.

\section{Materials and methods}

Reagents and cell culture. Res was purchased from Xi'an Hao-Xuan Biotechnology Co., Ltd. (Xi'an, China). HCT116 cell line was obtained from the American Type Culture Collection (ATCC, Manassas, VA, USA). All primary antibodies were purchased from Santa Cruz Biotechnology (Santa Cruz, CA, USA). PI3K inhibitor (LY294002) was purchased from Selleckchem (Houston, TX, USA). Cells were maintained in Dulbecco's modified Eagle's medium (DMEM) with $10 \%$ fetal bovine serum (FBS), penicillin $(100 \mathrm{U} / \mathrm{ml})$ and streptomycin $(100 \mu \mathrm{g} / \mathrm{ml})$ at $37^{\circ} \mathrm{C}$ in $5 \% \mathrm{CO}_{2}$.

Recombinant adenoviral constructs for BMP7, GFP, PTEN and SiRNA for PTEN. The recombinant adenoviral vectors were constructed following the AdEasy system (23). Briefly, the coding sequence (CDS) of human BMP7, green fluorescent protein (GFP) and PTEN were amplified, the siRNA fragments for PTEN knockdown was synthesized commercially. These fragments were cloned into the shuttle vector pAdTrace, respectively. Then, the shuttle vectors were linearized and transfected into HEK293 cells for the package of the recombinant adenoviruses, which were designated as AdBMP7, AdPTEN and AdsiPTEN. The recombinant adenoviruses mediated the overexpression were tagged with green GFP or red fluorescent protein (RFP) for knockdown to track the viruses, and the recombinant adenovirus expressing GFP (AdGFP) only was used as vehicle control.

Cell viability and proliferation assays. The cell viability was determined with cell counting kit-8 (CCK-8). In brief, HCT116 cells were seeded in 96-well plates with a density of $3 \times 10^{3}$ cells/well. Then, the cells were treated with different concentrations of Res, recombinant adenovirus or DMSO for 24, 48 and $72 \mathrm{~h}$. At the scheduled time-point, $10 \mu \mathrm{l}$ of CCK-8 was added into each well and incubated for $4 \mathrm{~h}$. The absorbance was determined at $450 \mathrm{~nm}$ with a micro-plate reader. Each test was conducted in triplicate.
Flow cytometric analysis for cell cycle and apoptosis. Cells were seeded in 6-well plates and treated with different concentrations of Res for $48 \mathrm{~h}$. For cell cycle analysis, cells were harvested and washed with phosphate-buffered saline (PBS, $\left.4^{\circ} \mathrm{C}\right)$, fixed with cold $\left(4^{\circ} \mathrm{C}\right) 70 \%$ ethanol, washed with 50 and $30 \%$ ethanol, and PBS. Finally, cells were stained with $1 \mathrm{ml}$ of propidium iodide (PI, $20 \mathrm{mg} / \mathrm{ml})$ containing RNase $(1 \mathrm{mg} / \mathrm{ml})$ in PBS for $30 \mathrm{~min}$, followed by flow cytometry analysis. For apoptosis analysis, cells were harvested and washed with PBS $\left(4^{\circ} \mathrm{C}\right)$, followed by incubating with Annexin V-EGFP (\#KGA104, KeyGen Biotech, Nanjing, China) and PI. Finally, the cells were analyzed with fluorescence activated cell sorting (FACS). For apoptosis stainging, cells were plated in 24-well plates and treated with different concentration of Res for $24 \mathrm{~h}$. Then the medium was discarded and the cells washed twice with PBS $\left(4^{\circ} \mathrm{C}\right)$, adding $500 \mu \mathrm{l}$ binding buffer and $5 \mu 1$ Annexin V-EGFP, followed by incubating for $10 \mathrm{~min}$. Finally, the buffer was discarded and cells washed with PBS $\left(4^{\circ} \mathrm{C}\right)$ carefully twice, then images were taken under a microscope. Each assay was done in triplicate.

Reverse transcription and polymerase chain reaction analysis (RT-PCR). Subconfluent HCT116 cells were plated in T25 flasks and treated with different concentrations of Res or DMSO. Total RNA was extracted with TRIzol reagent (Invitrogen, USA) and subjected to RT reaction to generate cDNA. Then, the cDNAs were used as templates for detecting the expression level of target genes with PCR. The primer sequences are available upon request. Each assay was carried out in triplicate.

Western blot assay. Subconfluent HCT116 cells were seeded in 6-well plates, and then treated with different concentrations of Res and/or combined with corresponding recombinant adenovirus. At the scheduled time-point, cells lysates were collected and boiled for $10 \mathrm{~min}$. All samples were subjected to electrophoresis with SDS-PAGE and transfered to polyvinylidene fluoride membranes, blotted with corresponding primary antibodies and secondary antibodies conjugated with horseradish peroxidase, successively. Finally, the target bands were developed with SuperSignal West Femto Substrate (\#34095, Thermo Scientific, IL, USA). Each assay was done in triplicate.

Statistical analysis. Microsoft Excel was employed to calculate the standard deviations. The differences between groups were analyzed with the Student's t-test.

\section{Results}

Effects of Res on the proliferation of HCT116 cells. First, we determined the effects of Res on the viability of colon cancer cells with Cell Counting Kit-8 (CCK-8). The result shows that Res decreases the growth of cancer cell lines in a concentration and time-dependent manner (Fig. 1A). The level of proliferating cell nuclear antigen (PCNA) is markedly increased in a concentration-dependent manner (Fig. 1B and C). Cell cycle analysis showed that Res can apparently arrest the cell cycle at $\mathrm{S}$ phase in HCT116 cells (Fig. 1D). These data confirmed that Res is able to suppress the proliferation of HCT116 cells. 
A

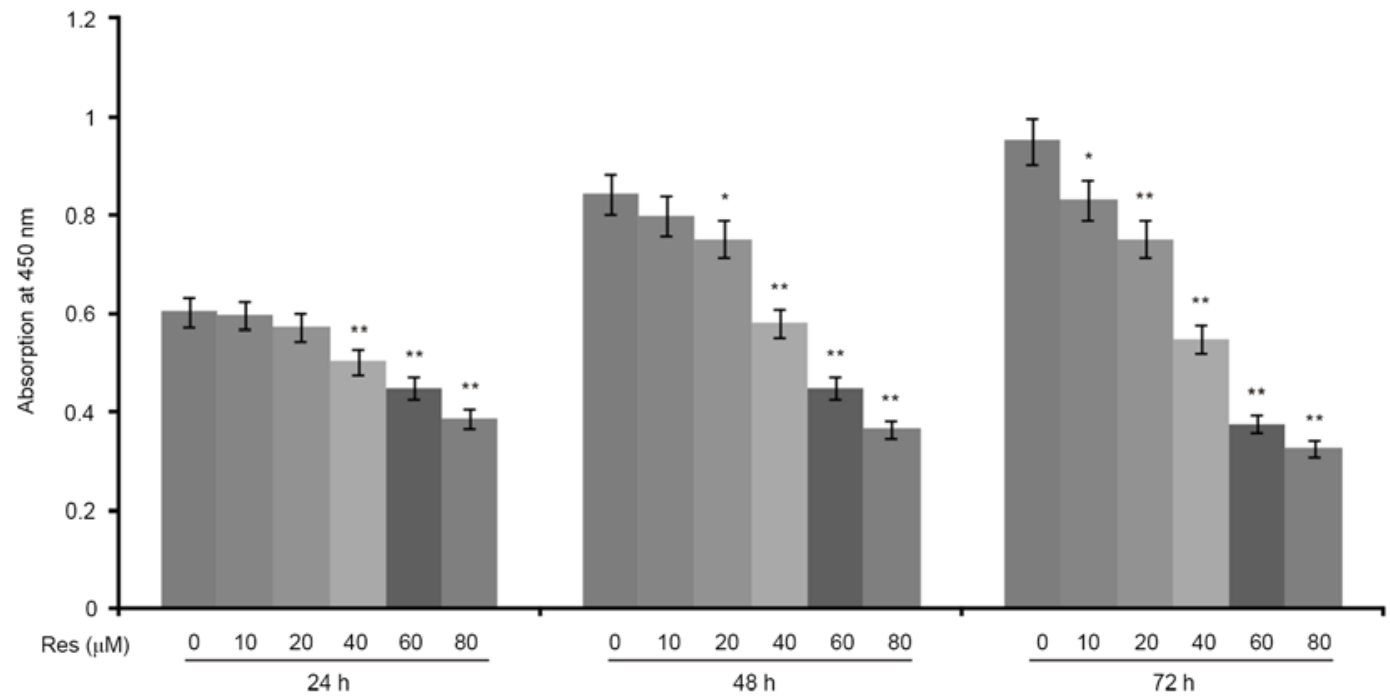

B
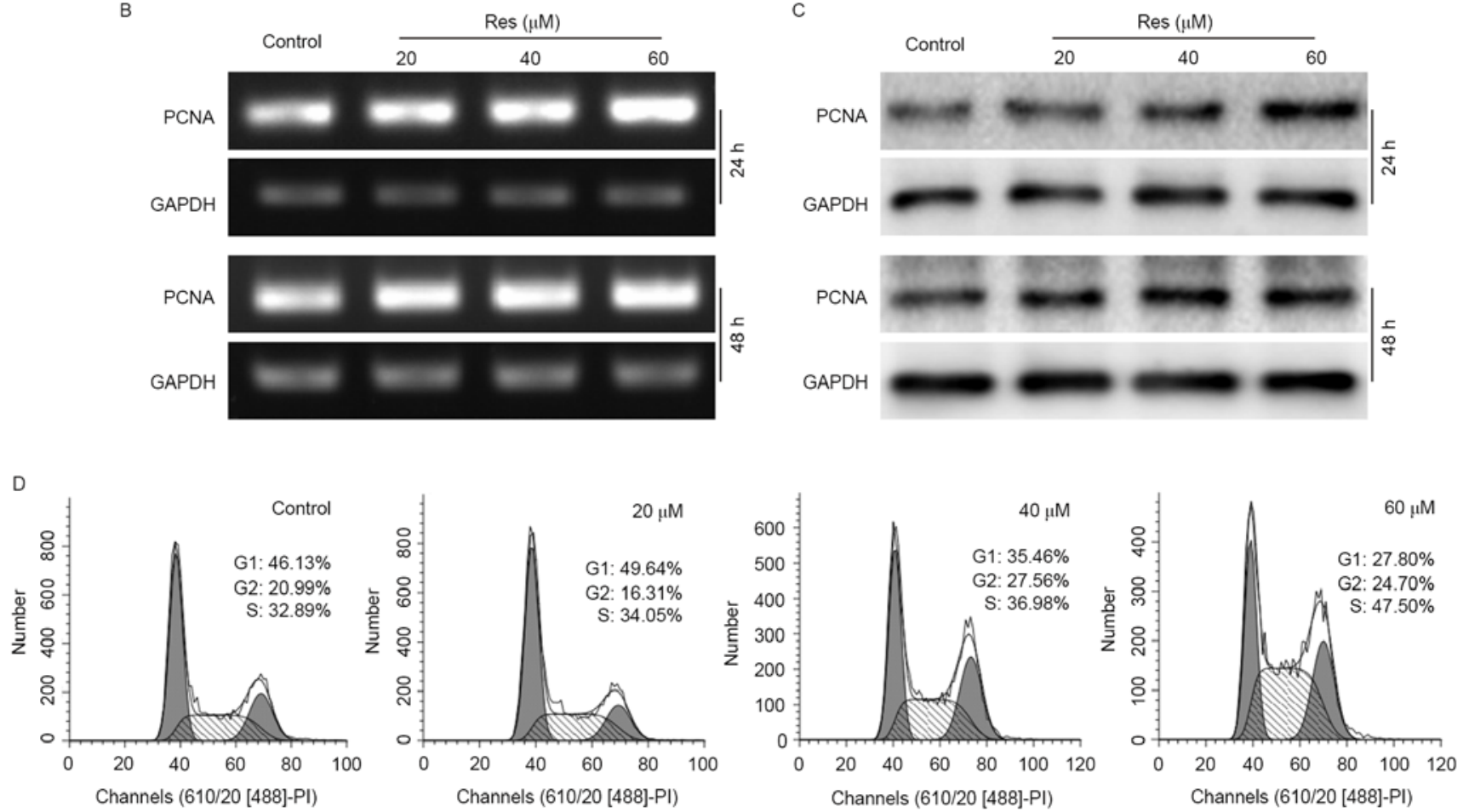

Figure 1. Effects of Res on the proliferation of HCT116 cells. (A) CCK-8 assay results showed the antiproliferation effect of Res on HCT116 cells ("p $<0.05$ vs control, ${ }^{* *}$ p $<0.01$ vs control). (B) PCR analysis results showed the effect of Res on the mRNA expression of PCNA in HCT116 cells. (C) Western blot analysis results showed the effect of Res on the protein level of PCNA in HCT116 cells, GAPDH was used as loading control. (D) Flow cytometry analysis results showed the cell cycle arrest effect of Res in HCT116 cells.

Effects of Res on apoptosis-inducing in HCT116 cells. We next conducted further analyses to figure out whether Res can induce colon cancer cells to undergo apoptosis. Annexin V-EGFP staining, flow cytometry and western blot analysis are employed to evaluate the effect of Res on apoptosis in HCT116 cells. The results showed that Res can induce apoptosis clearly and concentration dependently. Western blot assay showed that Res increases the level of Bad, but markedly decreases the level of Bcl-2 prominently in a concentration-dependent manner (Fig. 2A). The flow cytometory analysis results showed that Res can greatly increase the percentage of apoptotic cells (Fig. 2B). The Annexin V-EGFP staining results also showed that Res can apparently induce apoptosis in HCT116 cells (Fig. 2C).
These results strongly suggested that Res is an effective apoptosis inducer for human colon cancer cells.

Effects of Res on the expression of BMP7 in HCT116 cells. Although Res may be a powerful anticancer agent, the specific mechanism underlying this process remains unclear. Hence, we attempted to unveil the possible mechanism for this anticancer activity of Res. The western blot assay results showed that BMP7 is detectable in the available colon cancer cell lines and FHC cells, but the level of BMP7 in colon cancer cells is higher than that of FHC cells (Fig. 3A). This implied that BMP7 may be associated with colon cancer. The PCR and western blot assay results showed that Res greatly upregulates 


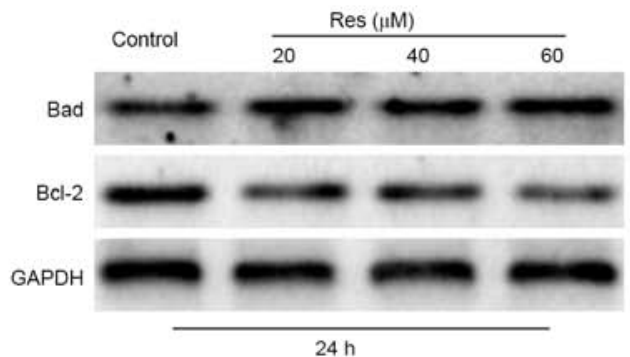

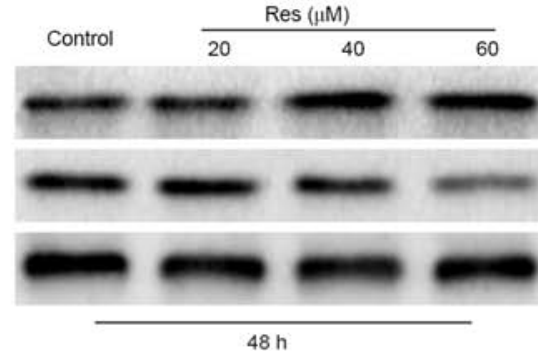
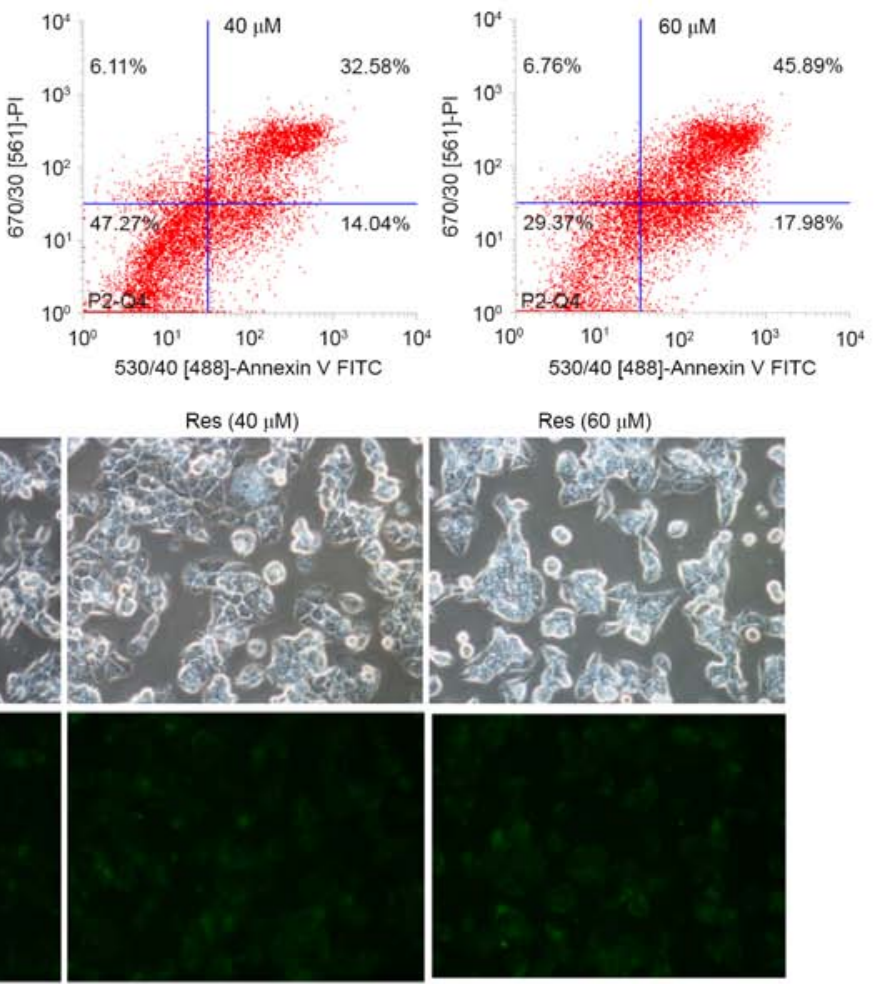

Figure 2. Effects of Res on induction of apoptosis in HCT116 cells. (A) Western blot analysis results showed the effect of Res on the protein level of Bad and Bcl-2 in HCT116 cells, GAPDH was used as loading control. (B) Flow cytometry analysis results showed the effect of Res on apoptosis in HCT116 cells. (C) Annexin V EGFP staining results showed the effect of Res on apoptosis in HCT116 cells.

A

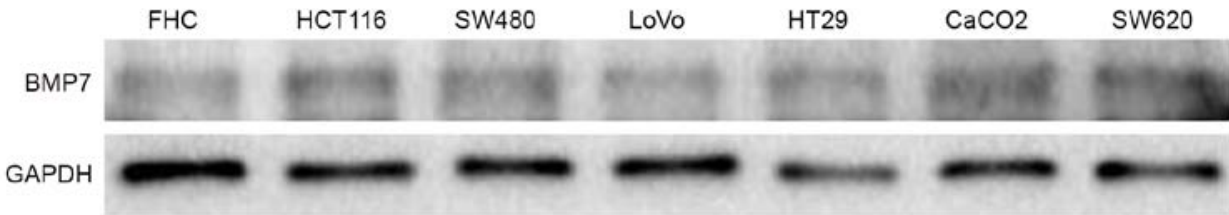

B

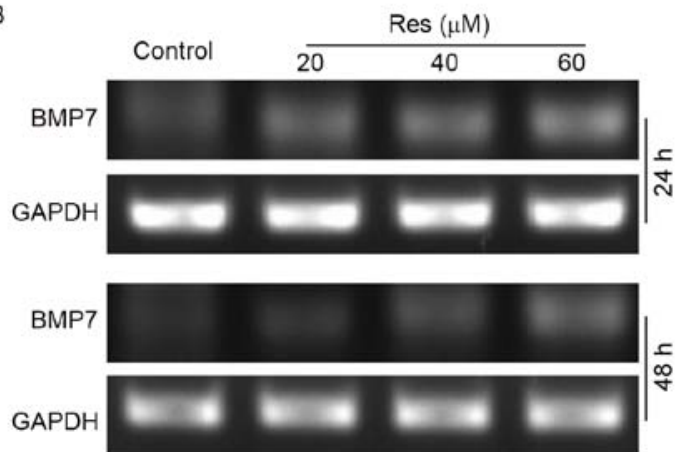

$\mathrm{C}$

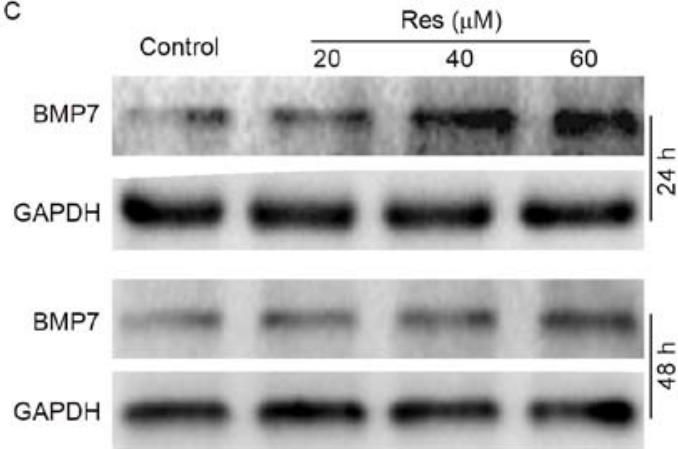

Figure 3. Effects of Res on the expression of BMP7 in HCT116 cells. (A) Western blot analysis showed the endogenous expression of BMP7 in colon cancer cells and FHC cells, GAPDH was used as loading control. (B) PCR analysis showed the effect of Res on the mRNA expression of BMP7 in HCT116 cells. (C) Western blot analysis showed the effect of Res on the protein level of BMP7 in HCT116 cells, GAPDH was used as loading control. 

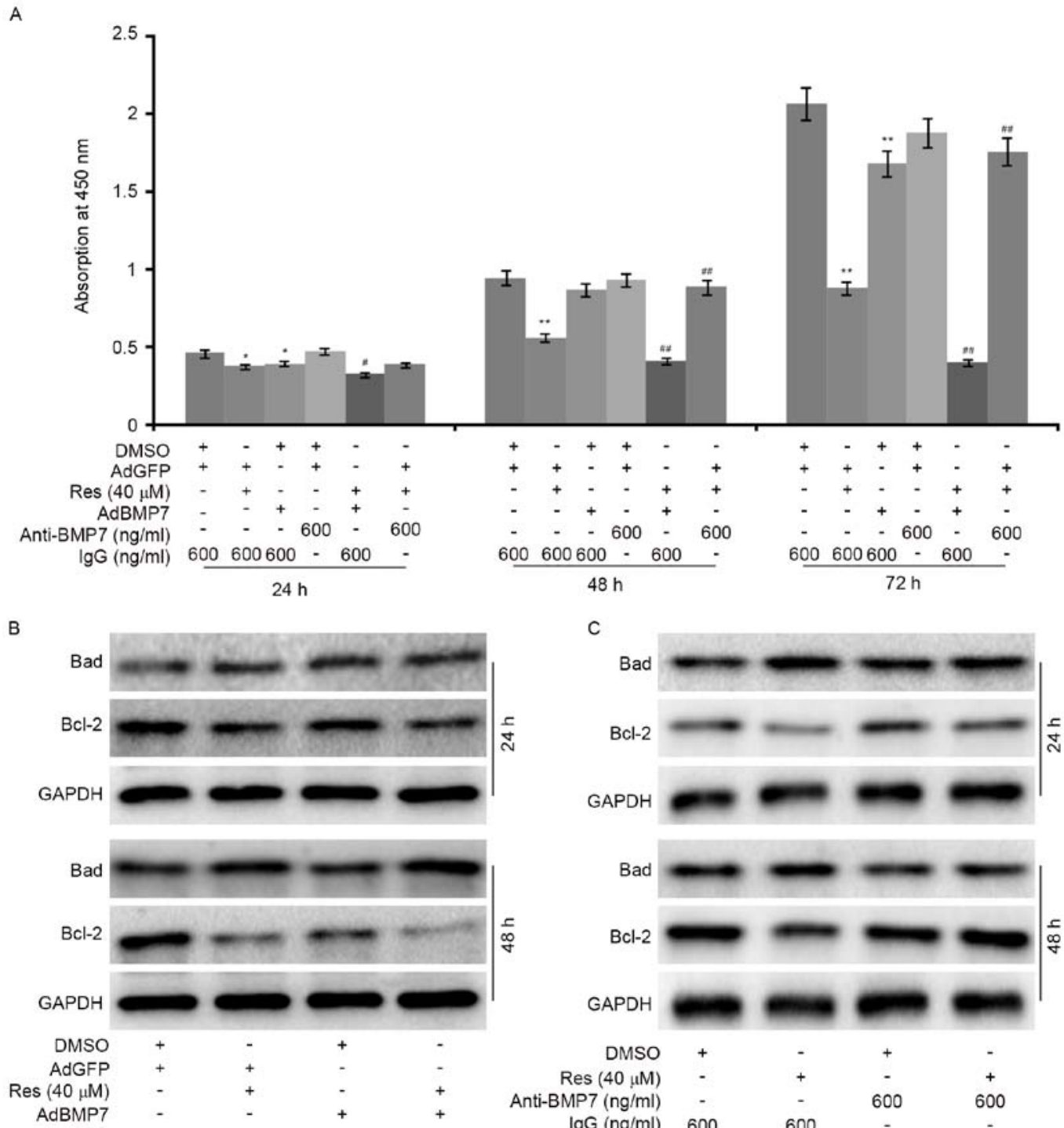

Figure 4. Effects of BMP7 on the anticancer activity of Res in HCT116 cells. (A) CCK-8 assay results showed the effect BMP7 or BMP7 specific antibody on the antiproliferation effect of Res on HCT116 cells ("p $<0.05$ vs control, ${ }^{* *} \mathrm{p}<0.01$ vs control; ${ }^{\#} \mathrm{p}<0.05$ vs Res group, ${ }^{\# \#} \mathrm{p}<0.01$ vs Res group). (B) Western blot analysis results showed the effect of BMP7 on the protein level of Bad and Bcl-2 affected by Res in HCT116 cells. GAPDH was used as loading control. (C) Western blot assay showed the effect of BMP7 specific antibody on the protein level of Bad and Bcl-2 affected by Res in HCT116 cells, GAPDH was used as loading control.

BMP7 with a concentration and time-dependent manner (Fig. 3B and C). These data suggested that upregulation of BMP7 may be involved in the anticancer activity of Res in colon cancer.

Effects of BMP7 on the anticancer activity of Res in HCT116 cells. As Res can upregulate BMP7 in colon cancer cells, we next explored the impact of BMP7 on the anticancer effect of Res in colon cancer cells. The CCK- 8 assay results showed that exogenous expression of BMP7 can reduce the viability of cancer cells and markedly enhance the antiproliferative effect of Res; while the BMP7 antibody $(600 \mathrm{ng} / \mathrm{ml})$ almost reverse the antiproliferative effect of Res $(40 \mu \mathrm{M})$ in the HCT116 cells (Fig. 4A). Regarding apoptosis, BMP7 increases the protein level of Bad which was upregulated by Res, and potentiates the effect of Res on decreasing the level of Bcl-2 (Fig. 4B). However, the BMP7 antibody triggers a reversed phenotype for the level of Bad and Bcl-2 affected by Res (Fig. 4C). These results indicated that BMP7 may mediate the anticancer effect of Res in human colon cancer cells.

Effects of BMP7 on PI3K/Akt signaling affected by Res in HCT116 cells. As a member of BMPs, BMP7 exerts its function not only through the canonical BMPs/Smads pathway, but also the non-canonical BMPs/Smads pathway, such as PI3K/ Akt signaling (24). We evaluated the effect of BMP7 on these signalings in HCT116 cells. Western blot assay showed that Res exhibits no apparent effect on increasing the phosphorylation of Smad1/5/8 (Fig. 5A), but decreased the total level of $\mathrm{Akt} 1 / 2$ and the phosphorylation of $\mathrm{Akt1} / 2$ (p-Akt1/2/3) in a concentration-dependent manner (Fig. 5B). These results implied that the effect of BMP7 on the antiproliferative effect of Res may not be mediated through the canonical BMP/Smad signaling, but associated with the inactivation of PI3K/Akt signaling. Further analysis showed that exogenous expressed BMP7 potentiates the effect of Res on decreasing the level of 
A

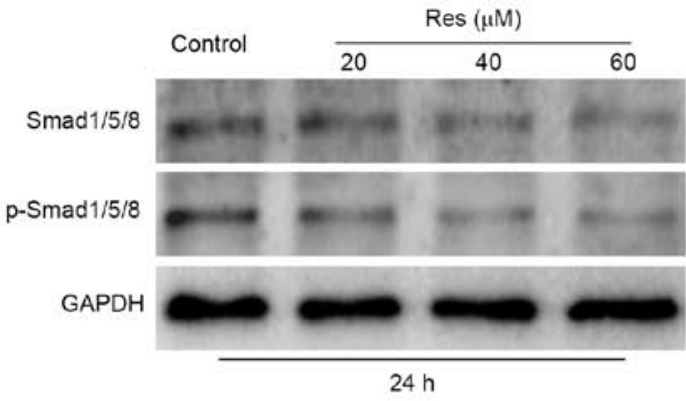

B

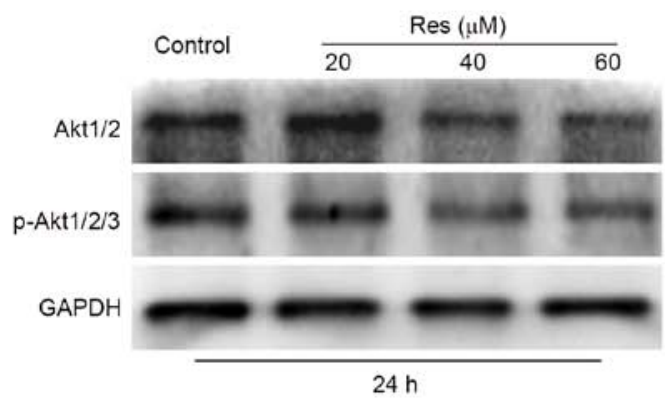

C

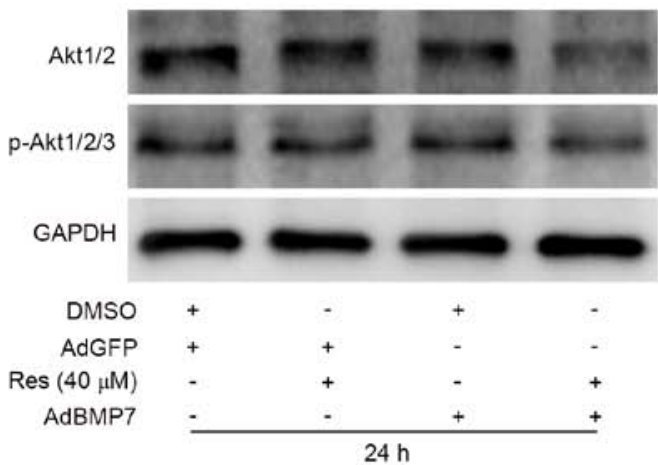

D

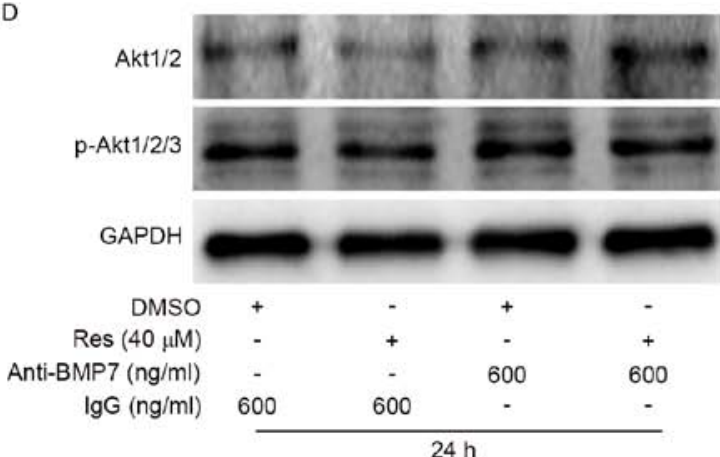

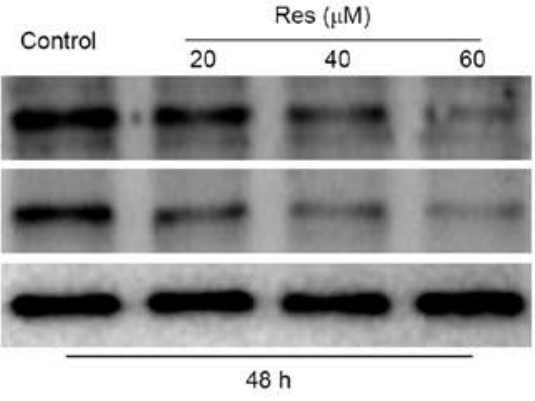
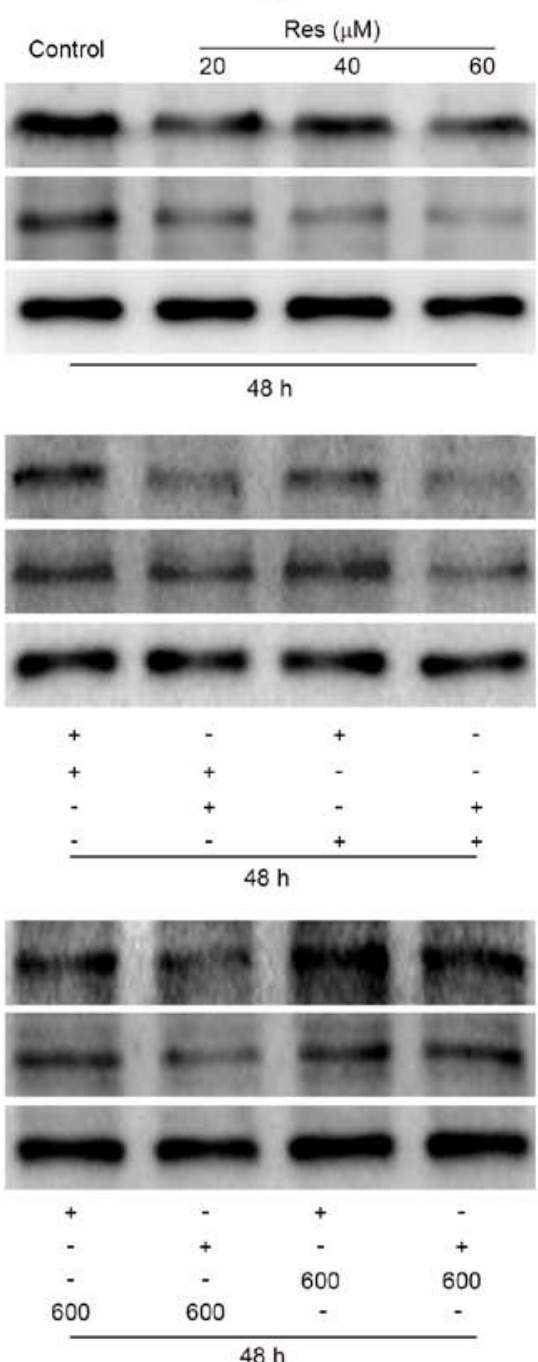

Figure 5. Effects of BMP7 on PI3K/Akt signaling affected by Res in HCT116 cells. (A) Western blot analysis showed the effect of Res on the protein level of Smad1/5/8 and p-Smad1/5/8, GAPDH was used as loading control. (B) Western blot analysis showed the effect of Res on the protein level of Akt1/2 and p-Akt1/2/3 in HCT116 cells, GAPDH was used as loading control. (C) Western blot analysis showed the effect of BMP7 on the protein level of Akt1/2 and p-Akt1/2/3 affected by Res in HCT116 cells, GAPDH was used as loading control. (D) Western blot analysis showed the effect of BMP7 specific antibody on the protein level of Akt1/2 and p-Akt1/2/3 affected by Res in HCT116 cells, GAPDH was used as loading control.

Akt1/2 and p-Akt1/2/3 (Fig. 5C), while BMP7 antibody apparently reverses these effects of Res in HCT116 cells (Fig. 5D). These results indicated that the anticancer activity of Res may contribute to the inhibition of PI3K/Akt signaling by upregulating BMP7 in HCT116 cells.

Effects of BMP7 on the phosphorylation of PTEN affected by Res in HCT116 cells. Since PTEN negatively regulates PI3K/Akt signaling and is often inactivated by mutations (including deletions) in a variety of cancer (25), we investi- gated whether PTEN is involved in the effect of BMP7 on the antiproliferative activity of Res. PCR analysis results showed that Res exerts no substantial effect on the mRNA expression of PTEN in HCT116 cells (Fig. 6A). The western blot assay results revealed that Res has no apparent effect on the total protein level of PTEN in HCT116 cells, but markedly decrease the phosphorylation of PTEN (Fig. 6B). Exogenous BMP7 can potentiate the effect of Res on decreasing the phosphorylation of PTEN (Fig. 6C), while BMP7 antibody can partly reverse the Res-induced decreasing phosporylation of PTEN (Fig. 6D). 
A

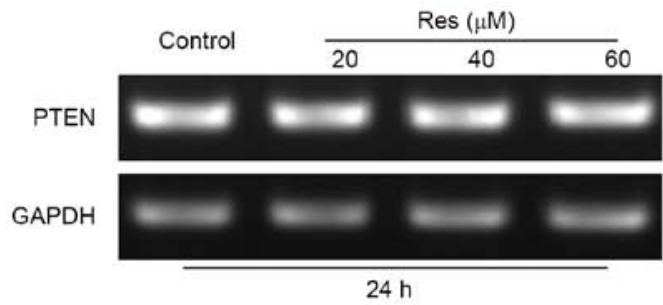

B

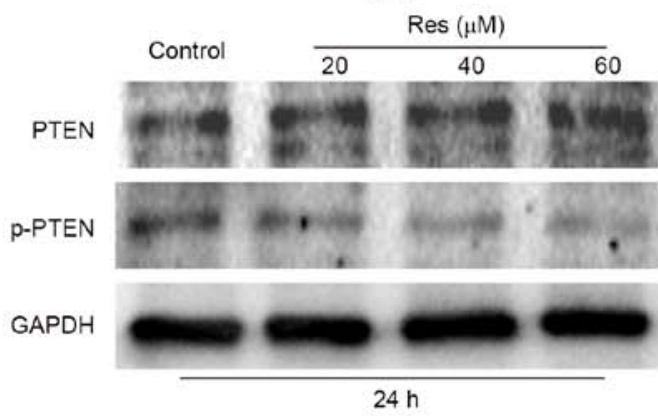

c

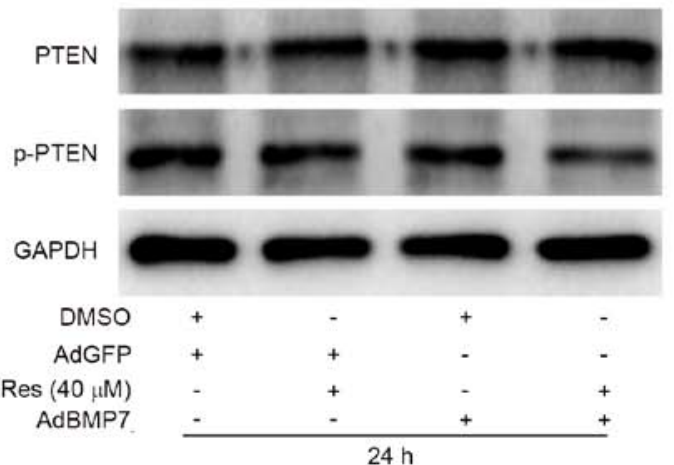

D

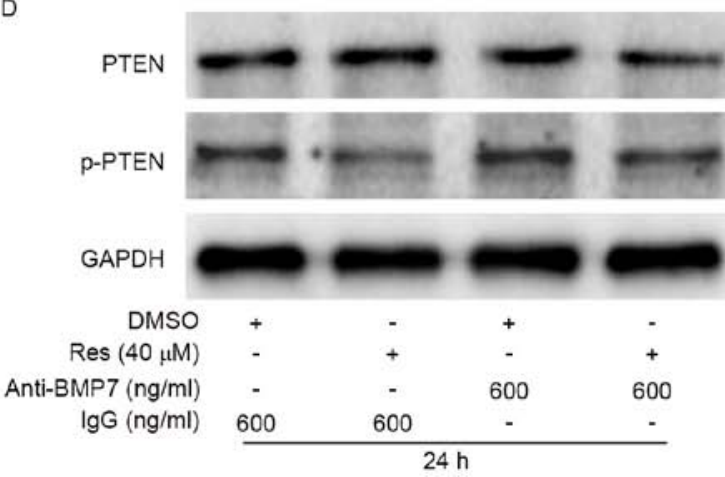

\begin{tabular}{cccc}
\multirow{2}{*}{ Control } & \multicolumn{3}{c}{$\operatorname{Res}(\mu \mathrm{M})$} \\
\cline { 2 - 3 } & 20 & 40 & 60 \\
\hline
\end{tabular}
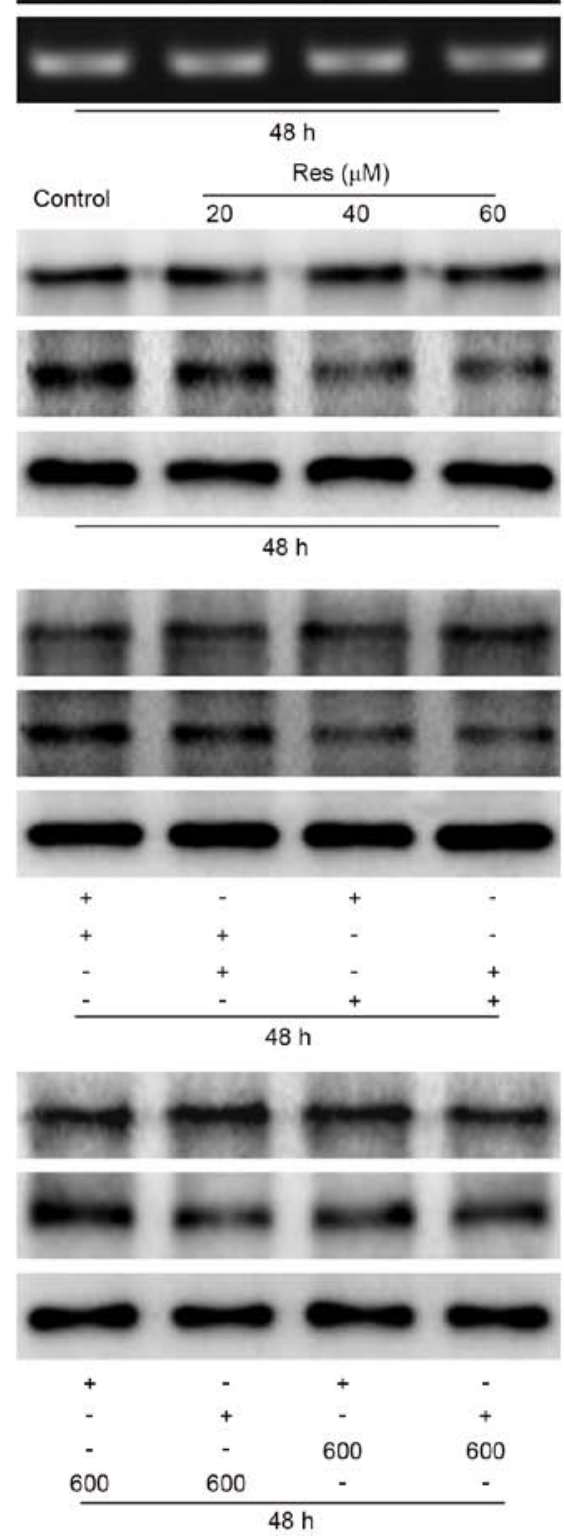

Figure 6. Effects of BMP7 on the phosphorylation of PTEN affected by Res in HCT116 cells. (A) PCR analysis showed the effect of Res on the mRNA expression of PTEN in HCT116 cells. (B) Western blot analysis showed the effect of Res on the protein level of PTEN and p-PTEN in HCT116 cells, GAPDH was used as loading control. (C) Western blot analysis showed the effect of BMP7 on the protein level of PTEN and p-PTEN affected by Res in HCT116 cells, GAPDH was used as loading control. (D) Western blot analysis showed the effect of BMP7 specific antibody on the protein level of PTEN and p-PTEN affected by Res in HCT116 cells, GAPDH was used as loading control.

These data suggested that BMP7 may mediate the anticancer activity of Res by suppressing the phosphorylation of PTEN in HCT116 cells.

\section{Discussion}

In this study, we investigated the anticancer activity of Res in HCT116 cells and dissected the possible mechanism underlying this process. We found that Res exhibits efficacious antiproliferation and apoptosis-inducing effect in HCT116 cells, which supported that it may be a plausible candidate for the treatment of human colon cancer cells. In terms of mechanism, we found that this anticancer activity of Res may be mediated by upregulating BMP7 to inactivate the PI3K/Akt signaling through partly suppressing the phosphorylation of PTEN.

Res is mainly used as a nutritional supplement in terms of its effects on cardiovascular protective (26), antiplatelet (27), antioxidant (28), antiinflammatory (29) and blood glucoselowering (30). Emerging evidence indicated that Res has been shown to suppress proliferation of various tumor cells, such as colon cancer (31), but the mechanism of anticancer activity for Res has not been completely elucidated yet. Our data confirmed Res can inhibit proliferation and induce apoptosis in HCT116 cells concentration dependently, which suggested 
that Res may be a potential natural product as chemotherapy drug or adjuvant for colon cancer treatment. As reported, several targets have been demonstrated to be associated with the anticancer activity of Res, such as the inhibition of TNF- $\alpha$ (32), p38 MAPK signaling (33) and PI3K/Akt signaling (12), but the specific molecular mechanism still need to be further thoroughly investigated.

BMPs are crucial cytokines belonging to the TGF- $\beta$ superfamily, which were first described by Ali and Brazil (34). The aberrant expression or function of BMPs is correlated with the proliferation, differentiation, and apoptosis of cancer cells, such as BMP2, BMP6 and BMP7 (17,19,35). Briefly, BMPs bind with two type receptors (type I and II) to form a complex, which then recruits and phosphorylates the receptor activated Smads (R-Smads). The activated R-Smads bind to the common Smad (Co-Smad, Smad4) to form complex, and then translocates to the nucleus along with a number of transcription factors to regulate the expression of target genes (13). BMPs can also activate the non-canonical Smad pathways, such as PI3K/Akt, mitogenactivated protein kinase (MAPK) and nuclear factor $\kappa \mathrm{B}$ $(\mathrm{NF}-\kappa \mathrm{B})(36-38)$. In the present study, we found that Res can increase the level of BMP7 in HCT116 cells, which implied that the anticancer activity of Res may arise from upregulating the expression of BMP7. Then, we introduced recombinant adenovirus of BMP7 and the BMP7 specific antibody through the proliferation and apoptosis assay to evaluate the effect of BMP7 in the anticancer activity of Res. Our data showed that exogenous expression of BMP7 boosts the antiproliferation effect of Res on HCT116 cells, while the BMP7 antibody attenuates this activity substantially. This finding suggested that BMP7 can partly mediate the anticancer activity of Res in HCT116 cells, although the mechanism remains unclear.

As Res upregulates BMP7 in HCT116 cells and BMP7 partly mediates the anticancer effect of Res, so we tried to elucidate how BMP7 exerts its function, through canonical BMPs/Smads or the non-canonical BMPs/Smads signaling. Our data showed that Res exhibits suppressing effect on the BMPs/Smads signaling instead of activation, which suggested that BMP7 may mediate the anticancer effect of Res through the non-canonical BMPs/Smads signaling pathway. PI3K/ Akt is one of the important non-canonical BMP/Smad signaling pathway which is overactivated in many cancers. Therefore, it has been targeted for many anticancer drugs in various cancers $(21,39)$. It has already been reported that Res can inhibit PI3K/Akt signaling (12), so we speculated that BMP7 may affect PI3K/Akt signaling in HCT116 cells. With western blot assay, we recaptured the effect of Res on suppressing the phosphorylation of Akt. Further investigations demonstrated that exogenous expression of BMP7 can potentiate the inactivation of PI3K/Akt signaling induced by Res, while BMP7 antibody almost reverses this effect of Res on PI3K/Akt signaling in HCT116 cells. This evidence supported that BMP7 may really mediate the anticancer activity of Res through inactivating PI3K/Akt signaling in HCT116 cells. However, this result is not in agreement with BMP7 activation of the PI3K/Akt signaling in osteocytes (40), which may dependent on the cell types and microenvironment or context. These data strongly revealed that the Res-induced inactivation of PI3K/Akt signaling may contribute to the upregulation of BMP7 in HCT116 cells.
PI3K/Akt signaling is one of the critical pathways for cell survival and often overactivated in various cancers $(39,41)$. This pathway can be regulated by many factors, such as activated by IGF and inactivated by PTEN $(42,43)$. PTEN acts as the essential endogenous negative modulator of PI3K/Akt signaling by dephosphorylating of the 3'-phosphate of the inositol ring in phosphatidylinositol $(3,4,5)$-trisphosphate $\left(\mathrm{PIP}_{3}\right)$ to form phosphatidylinositol (4,5)-biphosphate $\left(\mathrm{PIP}_{2}\right)$ (44). Since $\mathrm{PIP}_{3}$ is a primary activator of the signaling to intermediate the activation of Akt, the dephosphorylation of $\mathrm{PIP}_{3}$ by PTEN results in inhibition of the PI3K/Akt signaling. Based on this and the results discussed above, we speculated that the BMP7 mediated inactivation of PI3K/Akt signaling may be resulted from upregulating PTEN in HCT116 cells. Our results indicate that Res shows no substantial effect on the mRNA level of PTEN in HCT116 cells, or the total protein level of PTEN. However, Res can apparently decrease the phosphorylation of PTEN, which is consistent with the previous studies that PTEN is involved in the anticancer effect of Res in cancer cells (45-47). To further unveil the relationship between BMP7 and PTEN, we introduced the BMP7 recombinant adenovirus and a specific antibody. The results showed that exogenous expression of BMP7 enhances the ability of Res to reduce the phosphorylation of PTEN, while the immunodepletion of BMP7 with a specific antibody apparently reverses the Res-induced decrease phosphorylation of PTEN in HCT116 cells. Taken together, these data demonstrated that BMP7 may mediate the Res-induced inactivation of PI3K/Akt signaling through decreasing phosphorylation of PTEN in HCT116 cells.

In conclusion, these data demonstrated that Res may be used as an effective chemotherapy drug for human colon cancer. The anticancer activity of Res in colon cancer may be mediated by inactivating the PI3K/Akt signaling, which may be the result of the Res-induced upregulation of BMP7 to suppress the phosphorylation of PTEN.

\section{Acknowledgements}

We thank Professor T.C. He (Medical Center of University of Chicago, Chicago IL, USA) for his kind provision of the recombinant adenoviruses. This study was supported in part by the National Natural Science Foundation of China (grant nos. NSFC 81372120, 81572226 to Bai-Cheng He).

\section{References}

1. Brenner H, Kloor M and Pox CP: Colorectal cancer. Lancet 383: 1490-1502, 2014

2. Tran NH, Cavalcante LL, Lubner SJ, Mulkerin DL, LoConte NK, Clipson L, Matkowskyj KA and Deming DA: Precision medicine in colorectal cancer: The molecular profile alters treatment strategies. Ther Adv Med Oncol 7: 252-262, 2015.

3. Brenner H, Bouvier AM, Foschi R, Hackl M, Larsen IK, Lemmens V, Mangone L and Francisci S; EUROCARE Working Group: Progress in colorectal cancer survival in Europe from the late 1980 s to the early 21 st century: The EUROCARE study. Int J Cancer 131: 1649-1658, 2012.

4. Sankaranarayanan R, Swaminathan R, Brenner H, Chen K, Chia KS, Chen JG, Law SC, Ahn YO, Xiang YB, Yeole BB, et al: Cancer survival in Africa, Asia, and Central America: A population-based study. Lancet Oncol 11: 165-173, 2010.

5. Wang X, Tanaka M, Krstin S, Peixoto HS, Moura CC and Wink M: Cytoskeletal interference - A new mode of action for the anticancer drugs camptothecin and topotecan. Eur J Pharmacol 789: 265-274, 2016. 
6. Song XL, Liu S, Jiang Y, Gu LY, Xiao Y, Wang X, Cheng L and Li XT: Targeting vincristine plus tetrandrine liposomes modified with DSPE-PEG2000-transferrin in treatment of brain glioma. Eur J Pharm Sci 96: 129-140, 2017.

7. Noh JK, Naeem M, Cao J, Lee EH, Kim MS, Jung Y and Yoo JW: Herceptin-functionalized pure paclitaxel nanocrystals for enhanced delivery to HER2-postive breast cancer cells. Int J Pharm 513: 543-553, 2016.

8. Frémont L: Biological effects of resveratrol. Life Sci 66: 663-673, 2000.

9. Orallo F: Comparative studies of the antioxidant effects of cisand trans-resveratrol. Curr Med Chem 13: 87-98, 2006.

10. Zhu M, Zhang Q, Wang X, Kang L, Yang Y, Liu Y, Yang L, Li J, Yang L, Liu J, et al: Metformin potentiates anti-tumor effect of resveratrol on pancreatic cancer by down-regulation of VEGF-B signaling pathway. Oncotarget 7: 84190-84200, 2016

11. Chin YT, Hsieh MT, Yang SH, Tsai PW, Wang SH, Wang CC, Lee YS, Cheng GY, HuangFu WC, London D, et al: Antiproliferative and gene expression actions of resveratrol in breast cancer cells in vitro. Oncotarget 5: 12891-12907, 2014

12. Liu YZ, Wu K, Huang J, Liu Y, Wang X, Meng ZJ, Yuan SX, Wang DX, Luo JY, Zuo GW, et al: The PTEN/PI3K/Akt and $\mathrm{Wnt} / \beta$-catenin signaling pathways are involved in the inhibitory effect of resveratrol on human colon cancer cell proliferation. Int J Oncol 45: 104-112, 2014.

13. Bragdon B, Moseychuk O, Saldanha S, King D, Julian J and Nohe A: Bone morphogenetic proteins: A critical review. Cell Signal 23: 609-620, 2011 .

14. Zhang L, Ye Y, Long X, Xiao P, Ren X and Yu J: BMP signaling and its paradoxical effects in tumorigenesis and dissemination. Oncotarget 7: 78206-78218, 2016.

15. Herrera B, García-Álvaro M, Cruz S, Walsh P, Fernández M, Roncero C, Fabregat I, Sánchez A and Inman GJ: BMP9 is a proliferative and survival factor for human hepatocellular carcinoma cells. PLoS One 8: e69535, 2013.

16. Peng J, Yoshioka Y, Mandai M, Matsumura N, Baba T, Yamaguchi K, Hamanishi J, Kharma B, Murakami R, Abiko K, et al: The BMP signaling pathway leads to enhanced proliferation in serous ovarian cancer-A potential therapeutic target. Mol Carcinog 55: 335-345, 2016.

17. Wang L, Park P, Zhang H, La Marca F, Claeson A, Valdivia J and Lin CY: BMP-2 inhibits the tumorigenicity of cancer stem cells in human osteosarcoma OS99-1 cell line. Cancer Biol Ther 11: 457-463, 2011

18. Ying X, Sun Y and He P: Bone morphogenetic protein-7 inhibits EMT-associated genes in breast cancer. Cell Physiol Biochem 37: 1271-1278, 2015

19. Ren CM, Li Y, Chen QZ, Zeng YH, Shao Y, Wu QX, Yuan SX, Yang JQ, Yu Y, Wu K, et al: Oridonin inhibits the proliferation of human colon cancer cells by upregulating BMP7 to activate p38 MAPK. Oncol Rep 35: 2691-2698, 2016.

20. Cassar L, Nicholls C, Pinto AR, Chen R, Wang L, Li H and Liu JP: TGF-beta receptor mediated telomerase inhibition, telomere shortening and breast cancer cell senescence. Protein Cell 8: 39-54, 2017.

21. Leinhäuser I, Richter A, Lee M, Höfig I, Anastasov N, Fend F, Ercolino T, Mannelli M, Gimenez-Roqueplo AP, Robledo M, et al: Oncogenic features of the bone morphogenic protein 7 (BMP7) in pheochromocytoma. Oncotarget 6: 39111-39126, 2015.

22. Fresno Vara JA, Casado E, de Castro J, Cejas P, Belda-Iniesta C and González-Barón M: PI3K/Akt signalling pathway and cancer. Cancer Treat Rev 30: 193-204, 2004.

23. Luo J, Deng ZL, Luo X, Tang N, Song WX, Chen J, Sharff KA, Luu HH, Haydon RC, Kinzler KW, et al: A protocol for rapid generation of recombinant adenoviruses using the AdEasy system. Nat Protoc 2: 1236-1247, 2007.

24. Tate CM, Mc Entire J, Pallini R, Vakana E, Wyss L, Blosser W, Ricci-Vitiani L, D'Alessandris QG, Morgante L, Giannetti S, et al: A BMP7 variant inhibits tumor angiogenesis in vitro and in vivo through direct modulation of endothelial cell biology. PLoS One 10: e0125697, 2015.

25. Mendes RD, Canté-Barrett K, Pieters R and Meijerink JP: The relevance of PTEN-AKT in relation to NOTCH1-directed treatment strategies in T-cell acute lymphoblastic leukemia. Haematologica 101: 1010-1017, 2016.
26. Shen M, Jia GL, Wang YM and Ma H: Cardioprotective effect of resvaratrol pretreatment on myocardial ischemia-reperfusion induced injury in rats. Vascul Pharmacol 45: 122-126, 2006.

27. Lin KH, Hsiao G, Shih CM, Chou DS and Sheu JR: Mechanisms of resveratrol-induced platelet apoptosis. Cardiovasc Res 83: 575-585, 2009.

28. Valdecantos MP, Pérez-Matute P, Quintero P and Martínez JA Vitamin $\mathrm{C}$, resveratrol and lipoic acid actions on isolated rat liver mitochondria: All antioxidants but different. Redox Rep 15 207-216, 2010.

29. de la Lastra CA and Villegas I: Resveratrol as an antioxidant and pro-oxidant agent: Mechanisms and clinical implications. Biochem Soc Trans 35: 1156-1160, 2007.

30. Sadi G, Bozan D and Yildiz HB: Redox regulation of antioxidant enzymes: Post-translational modulation of catalase and glutathione peroxidase activity by resveratrol in diabetic rat liver. Mol Cell Biochem 393: 111-122, 2014

31. Varoni EM, Lo Faro AF, Sharifi-Rad J and Iriti M: Anticancer molecular mechanisms of resveratrol. Front Nutr 3: 8, 2016.

32. Bickenbach KA, Veerapong J, Shao MY, Mauceri HJ, Posner MC, Kron SJ and Weichselbaum RR: Resveratrol is an effective inducer of CArG-driven TNF-alpha gene therapy. Cancer Gene Ther 15: 133-139, 2008

33. Yuan SX, Wang DX, Wu QX, Ren CM, Li Y, Chen QZ, Zeng YH, Shao Y, Yang JQ, Bai Y, et al: BMP9/p38 MAPK is essential for the antiproliferative effect of resveratrol on human colon cancer. Oncol Rep 35: 939-947, 2016.

34. Ali IH and Brazil DP: Bone morphogenetic proteins and their antagonists: current and emerging clinical uses. Br J Pharmacol 171: 3620-3632, 2014.

35. Hu F, Zhang Y, Li M, Zhao L, Chen J, Yang S and Zhang X: BMP-6 inhibits the metastasis of MDA-MB-231 breast cancer cells by regulating MMP-1 expression. Oncol Rep 35: 1823-1830, 2016.

36. OrtuñoMJ,Ruiz-Gaspà S, Rodríguez-CarballoE,Susperregui AR, Bartrons R, Rosa JL and Ventura F: p38 regulates expression of osteoblast-specific genes by phosphorylation of osterix. J Biol Chem 285: 31985-31994, 2010.

37. Sánchez-Duffhues G, Hiepen C, Knaus P and Ten Dijke P: Bone morphogenetic protein signaling in bone homeostasis. Bone 80 43-59, 2015

38. Tang Y, Xie H, Chen J, Geng L, Chen H, Li X, Hou Y, Lu L, Shi S, Zeng X, et al: Activated NF- $\kappa \mathrm{B}$ in bone marrow mesenchymal stem cells from systemic lupus erythematosus patients inhibits osteogenic differentiation through downregulating Smad signaling. Stem Cells Dev 22: 668-678, 2013.

39. Mundi PS, Sachdev J, McCourt C and Kalinsky K: AKT in cancer: New molecular insights and advances in drug development. Br J Clin Pharmacol 82: 943-956, 2016.

40. Wang Z and Guo J: Mechanical induction of BMP-7 in osteocyte blocks glucocorticoid-induced apoptosis through PI3K/AKT/ GSK3 $\beta$ pathway. Cell Biochem Biophys 67: 567-574, 2013.

41. Mayer IA and Arteaga CL: The PI3K/AKT pathway as a target for cancer treatment. Annu Rev Med 67: 11-28, 2016.

42. Carnero A and Paramio JM: The PTEN/PI3K/AKT pathway in vivo, cancer mouse models. Front Oncol 4: 252, 2014.

43. Hixon ML, Paccagnella L, Millham R, Perez-Olle R and Gualberto A: Development of inhibitors of the IGF-IR/PI3K Akt/mTOR pathway. Rev Recent Clin Trials 5: 189-208, 2010.

44. Milella M,Falcone I, Conciatori F, Cesta Incani U, Del Curatolo A, Inzerilli N, Nuzzo CM, Vaccaro V, Vari S, Cognetti F, et al: PTEN: Multiple functions in human malignant tumors. Front Oncol 5: 24, 2015.

45. Leipert J, Kässner F, Schuster S, Händel N, Körner A, Kiess W and Garten A: Resveratrol potentiates growth inhibitory effects of rapamycin in PTEN-deficient lipoma cells by suppressing p70S6 kinase activity. Nutr Cancer 68: 342-349, 2016.

46. Jing X, Cheng W, Wang S, Li P and He L: Resveratrol induces cell cycle arrest in human gastric cancer MGC803 cells via the PTEN-regulated PI3K/Akt signaling pathway. Oncol Rep 35: 472-478, 2016

47. Wang G, Dai F, Yu K, Jia Z, Zhang A, Huang Q, Kang C, Jiang H and $\mathrm{Pu} \mathrm{P}$ : Resveratrol inhibits glioma cell growth via targeting oncogenic microRNAs and multiple signaling pathways. Int $\mathbf{J}$ Oncol 46: 1739-1747, 2015. 